\title{
Solvability of \\ Two-Point Boundary Value Problems for Fourth-Order Nonlinear Differential Equations at Resonance
}

\author{
Yuji Liu and Weigao Ge
}

\begin{abstract}
Under a resonance condition involving a two-point boundary value problem for a fourth-order nonlinear differential equation, we show its solvability.

Keywords: Fourth-order differential equation, two-point boundary value problem, solvability of boundary value problem, resonance
\end{abstract}

AMS subject classification: Primary 34K20, secondary 92D25

\section{Introduction}

Let $f:[0,1] \times \mathbb{R}^{4}$ be a continuous function and $e \in L^{1}[0,1]$. We consider the fourth-order differential equation

$$
x^{(4)}(t)=f\left(t, x(t), x^{\prime}(t), x^{\prime \prime}(t), x^{\prime \prime \prime}(t)\right)+e(t) \quad(0<t<1)
$$

subject to the boundary value conditions

$$
x^{\prime}(0)=x^{\prime}(1)=x^{\prime \prime \prime}(0)=x^{\prime \prime \prime}(1)=0 .
$$

Boundary value problems of this form were used to understand the static equilibrium of an elastic beam supported by sliding clamps. We refer the

Both authors: Beijing Inst. Techn., Dept. Math., Beijing, 100081, P.R. China; the first author also: Hunan Inst. Techn., Dept. Appl. Math., Yueyang, 414000, P.R. China; Liuyuji888@sohu.com

The first author was supported by the Science Foundation of Educational Committee of Hunan Province and the second author by the National Natural Sciences Foundation of P.R. China

ISSN 0232-2064 / \$2.50 C Heldermann Verlag Berlin 
reader to [11, 12] and the references therein. For example, Gupta [12] studied the solvability of the boundary value problem

$$
\left.\begin{array}{l}
-y^{(4)}+g(t, y(t))=e(t) \quad(0<t<1) \\
=y^{\prime \prime \prime}(0)=y^{\prime \prime \prime}(\pi)=0
\end{array}\right\} .
$$

Since (2) implies that the linear operator $L x=x^{(4)}$ defined in a suitable Banach space is not invertible, we call (2) a resonance boundary value condition. There are many other papers concerning the existence of solutions or positive solutions of fourth-order differential equations subjected to different kind of non-resonance boundary value conditions (see $[1-6,8,10,13,14,16]$ and the references therein).

To the best of our knowledge, the solvability of boundary value problem (1) - (2) has not been studied till now. The purpose of this paper is to establish an existence result for problem (1) - (2). Our method is based on the coincidence degree theory of Mawhin.

Now, we briefly recall some notations and an abstract existence result. Let $X$ and $Y$ be Banach spaces, $L: \operatorname{dom} L \subset X \rightarrow Y$ be a Fredholm operator of index zero, $P: X \rightarrow X$ and $Q: Y \rightarrow Y$ be projectors such that

$$
\begin{aligned}
\operatorname{Im} P & =\operatorname{Ker} L \\
\operatorname{Ker} Q & =\operatorname{Im} L \\
X & =\operatorname{Ker} L+\operatorname{Ker} P \\
Y & =\operatorname{Im} L+\operatorname{Im} Q .
\end{aligned}
$$

It follows that the reduced operator

$$
\left.L\right|_{\text {dom } L \cap \operatorname{Ker} P}: \operatorname{dom} L \cap \operatorname{Ker} P \rightarrow \operatorname{Im} L
$$

is invertible. We denote the inverse of that map by $K_{p}$.

If $\Omega$ is an open bounded subset of $X$ and $\operatorname{dom} L \cap \Omega \neq \emptyset$, where $\emptyset$ denotes the empty set, the map $N: X \rightarrow Y$ will be called $L$-compact on $\bar{\Omega}$ if $Q N(\bar{\Omega})$ is bounded and the product map $K_{p}(I-Q) N: \bar{\Omega} \rightarrow X$ is compact. The facts we use are [15: Theorem 2.4] and [7: Theorem IV.13].

Theorem 1. Let $L$ be a Fredholm operator of index zero and let $N$ be $L$-compact on $\Omega$. Assume that the following conditions are satisfied:

(i) $L x \neq \lambda N x$ for every $(x, \lambda) \in[(\operatorname{dom} L / \operatorname{Ker} L) \cap \partial \Omega] \times(0,1)$.

(ii) $N x \notin \operatorname{Im} L$ for every $x \in \operatorname{Ker} L \cap \partial \Omega$.

(iii) $\operatorname{deg}\left(\left.\Lambda Q N\right|_{\operatorname{Ker} L}, \Omega \cap \operatorname{Ker} L, 0\right) \neq 0$, where $\Lambda: \operatorname{Im} L \rightarrow \operatorname{Ker} L$ is some isomorphism. 
Then the equation $L x=N x$ has at least one solution in $\operatorname{dom} L \cap \bar{\Omega}$.

We use the classical spaces $C^{3}[0,1]$ and $L^{1}[0,1]$. For $x \in C^{3}[0,1]$, we use the norms $\|x\|_{\infty}=\max _{t \in[0,1]}|x(t)|$ and

$$
\|x\|=\max \left\{\|x\|_{\infty},\left\|x^{\prime}\right\|_{\infty},\left\|x^{\prime \prime}\right\|_{\infty},\left\|x^{\prime \prime \prime}\right\|_{\infty}\right\}
$$

and denote the norm in $L^{1}[0,1]$ by $\|x\|_{1}$. We also use the Sobolev space $W^{4,1}(0,1)$ defined by

$$
W^{4,1}=\left\{x:[0,1] \rightarrow \mathbb{R} \mid x, x^{\prime}, x^{\prime \prime}, x^{\prime \prime \prime} \text { abs. cont., } x^{(4)} \in L^{1}[0,1]\right\}
$$

with its usual norm.

\section{Main results}

In this section, we shall prove the existence result for problem (1) - (2). Let $X=C^{3}[0,1]$ and $Y=L^{1}[0,1]$. Define $L$ to be the linear operator from $\operatorname{dom} L \subset X$ to $Y$ with

$$
\operatorname{dom} L=\left\{x \in W^{4,1}(0,1) \mid x^{\prime}(0)=x^{\prime}(1)=x^{\prime \prime \prime}(0)=x^{\prime \prime \prime}(1)=0\right\}
$$

and $(L x)(t)=x^{(4)}(t)$ for $x \in \operatorname{dom} L \cap X$, and we define $N$ to be the nonlinear operator from $X$ to $Y$ with

$$
(N x)(t)=f\left(t, x(t), x^{\prime}(t), x^{\prime \prime}(t), x^{\prime \prime \prime}(t)\right)+e(t) \quad(0<t<1)
$$

for $x \in X$. Thus problem (1) - (2) can be written as $L x=N x$. We note that if $x \in \operatorname{dom} L$, then $\|x\|=\max \left\{\|x\|_{\infty},\left\|x^{\prime \prime \prime}\right\|_{\infty}\right\}$, since $\left\|x^{\prime}\right\|_{\infty} \leq\left\|x^{\prime \prime}\right\|_{\infty} \leq\left\|x^{\prime \prime \prime}\right\|_{\infty}$.

Lemma 1. The following results hold:

(i) $\operatorname{Ker} L=\{x \in X: x(t)=c(0 \leq t \leq 1)$ for some $c \in \mathbb{R}\}$.

(ii) $\operatorname{Im} L=\left\{y \in Y: \int_{0}^{1} y(s) d s=0\right\}$.

(iii) $L$ is a Fredholm operator of index zero.

(iv) If $\Omega$ is an open bounded subset such that $\operatorname{dom} L \cap \Omega \neq \emptyset$, then $N$ is $L$-compact on $\bar{\Omega}$.

Proof. (i): For $x \in \operatorname{Ker} L$ we have $x^{4}(t)=0$, thus $x(t)=a t^{3}+b t^{2}+c t+d$. On the other hand, $x^{\prime}(0)=x^{\prime}(1)=x^{\prime \prime \prime}(0)=x^{\prime \prime \prime}(1)=0$ implies that $a=b=$ $c=0$. So $x(t)=d$ for $t \in[0,1]$. Again, if $x=d$, then $x \in \operatorname{Ker} L$. This completes the proof of assertion (i). 
(ii): For $y \in \operatorname{Im} L$ there is $x \in \operatorname{dom} L$ such that $x^{(4)}=y$. So

$$
x(t)=\int_{0}^{t} \frac{(t-s)^{3}}{6} y(s) d s+a t^{3}+b t^{2}+c t+d .
$$

Since $x^{\prime}(0)=x^{\prime}(1)=x^{\prime \prime \prime}(1)=x^{\prime \prime \prime}(0)=0$, we get $c=a=0$ and $\int_{0}^{1} y(s) d s=$ 0 . Thus $y \in\left\{y \in Y: \int_{0}^{1} y(s) d s=0\right\}$. On the other hand, if $y \in Y$ and $\int_{0}^{1} y(s) d s=0$, let

$$
x(t)=\int_{0}^{t} \frac{(t-s)^{3}}{6} y(s) d s-\frac{t^{2}}{2} \int_{0}^{1} \frac{(1-s)^{2}}{2} y(s) d s .
$$

Then $x \in X$ and $x^{\prime}(0)=x^{\prime}(1)=x^{\prime \prime \prime}(0)=x^{\prime \prime \prime}(1)=0$. This implies $y \in \operatorname{Im} L$, so assertion (ii) is valid.

(iii): Define the projector $Q: Y \rightarrow Y$ by

$$
Q y(t)=\int_{0}^{1} y(s) d s \quad(y \in Y) .
$$

It is easy to check that, for $y \in Y, y-Q y \in \operatorname{Im} L$. So $y=\operatorname{Im} L+R$, again $\operatorname{Im} L \cap R=\{0\}$, hence $Y=\operatorname{Im} L \oplus R$. Together with that $\operatorname{Im} L$ is closed, thus $L$ is a Fredholm operator of index zero.

(iv) Let $\Omega$ be an open bounded subset in $X$ such that $\Omega \cap \operatorname{dom} L \neq \Phi$. Define the projector $P: X \rightarrow X$ by $P(x)=x(0)$. Then the generalized inverse $K_{p}: \operatorname{Im} L \rightarrow \operatorname{dom} L \cap \operatorname{Ker} P$ of $L$ can be written as

$$
\left(K_{p} y\right)(t)=\int_{0}^{t} \frac{(t-s)^{3}}{6} y(s) d s-\frac{t^{2}}{2} \int_{0}^{1} \frac{(1-s)^{2}}{2} y(s) d s .
$$

In fact, for $y \in \operatorname{Im} L$ we have

$$
\left(L K_{p}\right) y(t)=L\left(\int_{0}^{t} \frac{(t-s)^{3}}{6} y(s) d s-\frac{t^{2}}{2} \int_{0}^{1} \frac{(1-s)^{2}}{2} y(s) d s\right)=y(t) .
$$

Further, for $x \in \operatorname{dom} L \cap \operatorname{Ker} P$ we have

$$
\begin{aligned}
\left(K_{p} L x\right)(t) & =K_{p}\left(x^{(4)}(t)\right) \\
& =\int_{0}^{t} \frac{(t-s)^{3}}{6} x^{(4)}(s) d s-\frac{t^{2}}{2} \int_{0}^{1} \frac{\left.(1-s)^{2}\right) x^{(4)}(s)}{2} d s \\
& =\frac{t^{3}}{6} x^{\prime \prime \prime}(0)+\frac{t^{2}}{2} x^{\prime \prime}(0)+t x^{\prime}(0)+x(t)-x(0)-\frac{t^{2}}{2} x^{\prime \prime}(0) \\
& =x(t) .
\end{aligned}
$$


This shows $K_{p}=\left(\left.L\right|_{\operatorname{dom} L \cap \operatorname{Ker} P}\right)^{-1}$. Furthermore, $X=\operatorname{Ker} L \bigoplus \operatorname{Ker} P$. In fact, for $x \in X, x(t)-x(0) \in \operatorname{Ker} P$, so $X=\operatorname{Ker} P+\operatorname{Ker} L$, and again $\operatorname{Ker} L \cap \operatorname{Ker} P=\{0\}$. Then $X=\operatorname{Ker} L \oplus \operatorname{Ker} P$. From (4) we find

$$
\begin{aligned}
\left\|K_{p} y\right\|_{\infty} & \leq \frac{1}{6}\|y\|_{1}+\frac{1}{4}\|y\|_{1}=\frac{5}{12}\|y\|_{1} \\
\left\|\left(K_{p} y\right)^{\prime}\right\|_{\infty} & \leq \frac{1}{2}\|y\|_{1}+\frac{1}{2}\|y\|_{1}=\|y\|_{1} \\
\left\|\left(K_{p} y\right)^{\prime \prime \prime}\right\|_{\infty} & =\left\|\int_{0}^{t} y(s) d s\right\|_{\infty} \leq\|y\|_{1} .
\end{aligned}
$$

Since $\left(K_{p} y\right)^{\prime}(0)=\left(K_{p} y\right)^{\prime}(1)=0$, there is $\xi \in(0,1)$ such that $\left(K_{p} y\right)^{\prime \prime}(\xi)=0$. Hence for $t \in(01)$ we have

$$
\begin{aligned}
\left|\left(K_{p} y\right)^{\prime \prime}(t)\right| & =\left|\left(K_{p} y\right)^{\prime \prime}(t)-\left(K_{p} y\right)^{\prime \prime}(\xi)\right| \\
& =\left|\left(K_{p} y\right)^{\prime \prime \prime}(\eta)(t-\xi)\right| \\
& \leq\left|\left(K_{p} y\right)^{\prime \prime \prime}(\eta)\right|
\end{aligned}
$$

for $\eta \in(t, \xi)$ or $\eta \in(\xi, t)$. So

$$
\left\|\left(K_{p} y\right)^{\prime \prime}\right\|_{\infty} \leq\left\|\left(K_{p} y\right)^{\prime \prime \prime}\right\|_{\infty} \leq\|y\|_{1} .
$$

It follows that $\left\|K_{p} y\right\| \leq\|y\|_{1}$ for $y \in Y$. It is easy to see that

$$
(Q N x)(t)=\int_{0}^{1}\left(f\left(s, x(s), x^{\prime}(s), x^{\prime \prime}(s), x^{\prime \prime \prime}(s)\right)+e(s)\right) d s
$$

and

$$
\begin{aligned}
K_{p}( & I-Q) N x(t) \\
= & \int_{0}^{t} \frac{(t-s)^{3}}{6}\left(f\left(s, x(s), x^{\prime}(s), x^{\prime \prime}(s), x^{\prime \prime \prime}(s) b i g\right)+e(s)\right) d s \\
& -\frac{t^{2}}{2} \int_{0}^{1} \frac{(1-s)^{2}}{2}\left(f\left(s, x(s), x^{\prime}(s), x^{\prime \prime}(s), x^{\prime \prime \prime}(s)\right)+e(s)\right) d s \\
& -\left(\frac{t^{4}}{24}+\frac{t^{2}}{12}\right) \int_{0}^{1}\left(f\left(s, x(s), x^{\prime}(s), x^{\prime \prime}(s), x^{\prime \prime \prime}(s)\right)+e(s)\right) d s .
\end{aligned}
$$

By using the Ascoli-Arzela theorem, we can prove that $Q N(\bar{\Omega})$ is bounded and $K_{p}(I-Q) N: \bar{\Omega} \rightarrow X$ is compact. So $N$ is $L$-compact on $\bar{\Omega}$ 
Theorem 2. Let $f:[0,1] \times R^{4} \rightarrow R$ be a continuous function. Assume the following:

$\left(\mathbf{A}_{1}\right)$ There exist functions $a, b, c, d, g:[0,1] \rightarrow \mathbb{R}$ and $r \in L^{1}[0,1]$ and $a$ constant $\theta \in[0,1)$ such that

$|f(t, x, y, z, w)| \leq a(t)|x|+b(t)|y|+c(t)|z|+d(t)|w|+g(t)|w|^{\theta}+r(t) ;$

for all $t \in[0,1]$.

$\left(\mathbf{A}_{2}\right)$ There exists a constant $M>0$ such that if $|w|>M$, then

$$
|f(t, x, y, z, w)|>-\bar{\alpha}|x|+\bar{\beta}|w|-L_{1}
$$

for all $x, y, z \in R$ and $t \in[0,1]$, where $\bar{\beta}>\bar{\alpha}>0$ and $L_{1}>0$ are some constants.

$\left(\mathbf{A}_{3}\right)$ There is a constant $M_{1}>0$ such that if $|x(t)|>M_{1}$ for all $t \in[0,1]$, then

$$
\int_{0}^{1}\left(f\left(s, x(s), x^{\prime}(s), x^{\prime \prime}(s), x^{\prime \prime \prime}(s)\right)+e(s)\right) d s \neq 0 .
$$

$\left(\mathbf{A}_{4}\right) \lim _{|c| \rightarrow \infty} \frac{|f(t, c, 0,0,0)|}{|c|} \in(0,+\infty)$.

$\left(\mathbf{A}_{5}\right)$ There is a constant $M_{2}>0$ such that if $|c|>M_{2}$, then

$$
c f(t, c, 0,0,0)\left\{\begin{array}{l}
\leq 0 \\
o r \\
\geq 0
\end{array} \quad(0 \leq t \leq 1) .\right.
$$

$\left(\mathbf{A}_{6}\right)\|a\|_{1}+\|b\|_{1}+\|c\|_{1}+\|d\|_{1}<\frac{1}{2}\left(1-\frac{\bar{\alpha}}{\bar{\beta}}\right)$.

Then for every $e \in L^{1}[0,1]$ problem $(1)-(2)$ has at least one solution in $C^{3}[0,1]$.

Proof. Let

$$
\Omega_{1}=\{x \in \operatorname{dom} L / \operatorname{Ker} L: L x=\lambda N x \text { for some } \lambda \in(0,1)\} .
$$

If $x \in \Omega_{1}$, then $x \notin \operatorname{Ker} L, \lambda \neq 0$ and $N x \in \operatorname{Im} L$, thus $Q N x=0$, i.e.

$$
\begin{aligned}
& x^{(4)}(t)=\lambda f\left(t, x(t), x^{\prime}(t), x^{\prime \prime}(t), x^{\prime \prime \prime}(t)\right)+e(t) \quad(t \in[0,1]) \\
& x^{\prime}(0)=x^{\prime}(1)=x^{\prime \prime \prime}(0)=x^{\prime \prime \prime}(1)=0 \\
& \int_{0}^{1}\left(f\left(s, x(s), x^{\prime}(s), x^{\prime \prime}(s), x^{\prime \prime \prime}(s)\right)+e(s)\right) d s=0 .
\end{aligned}
$$


So there is $t_{1} \in(0,1)$ such that

$$
f\left(t_{1}, x\left(t_{1}\right), x^{\prime}\left(t_{1}\right), x^{\prime \prime}\left(t_{1}\right), x^{\prime \prime \prime}\left(t_{1}\right)\right)=-\int_{0}^{1} e(s) d s .
$$

This yields

$$
\left|f\left(t_{1}, x\left(t_{1}\right), x^{\prime}\left(t_{1}\right), x^{\prime \prime}\left(t_{1}\right), x^{\prime \prime \prime}\left(t_{1}\right)\right)\right| \leq\|e\|_{1} .
$$

Again, if $x \in \operatorname{dom} L$, then $(I-P) x \in \operatorname{dom} L \cap \operatorname{Ker} P$ and $L P x=0$. Thus, from Lemma 1,

$$
\|(I-P) x\|=\left\|K_{p} L(I-P) x\right\| \leq\|L(I-P) x\|_{1}=\|L x\|_{1} \leq\|N x\|_{1} .
$$

We consider two cases.

Case 1: $\left|x^{\prime \prime \prime}\left(t^{*}\right)\right| \leq M$ for some $t^{*} \in[0,1]$. In this case we have

$$
\left|x^{\prime \prime \prime}(t)\right|=\left|x^{\prime \prime \prime}\left(t^{*}\right)\right|+\left|\int_{t}^{t^{*}} x^{(4)}(s) d s\right| \leq M+\|L x\|_{1} \leq M+\|N x\|_{1} .
$$

Since $x^{\prime}(0)=x^{\prime}(1)=x^{\prime \prime \prime}(0)=x^{\prime \prime \prime}(1)=0$, there is $\xi \in(0,1)$ such that $x^{\prime \prime}(\xi)=0$, thus

$$
\left|x^{\prime \prime}(t)\right|=\left|x^{\prime \prime}(t)-x^{\prime \prime}(\xi)\right|=\left|x^{\prime \prime \prime}(\eta)(t-\xi)\right| \leq M+\|N x\|_{1} .
$$

Also, there is $\eta_{1} \in[0,1]$ such that

$$
\left|x^{\prime}(t)\right|=\left|x^{\prime}(t)-x^{\prime}(0)\right|=\left|x^{\prime \prime}\left(\eta_{1}\right) t\right| \leq M+\|N x\|_{1} .
$$

We claim that there is a $t^{* *} \in(0,1)$ such that $\left|x\left(t^{* *}\right)\right| \leq M_{1}$. Otherwise, if $|x(t)|>M_{1}$ for all $t \in[0,1]$, condition $\left(\mathrm{A}_{3}\right)$ implies

$$
\int_{0}^{1}\left(f\left(s, x(s), x^{\prime}(s), x^{\prime \prime}(s), x^{\prime \prime \prime}(s)\right)+e(s)\right) d s \neq 0 .
$$

On the other hand, since $L x \in \operatorname{Im} L$, we have

$$
\int_{0}^{1}\left(f\left(s, x(s), x^{\prime}(s), x^{\prime \prime}(s), x^{\prime \prime \prime}(s)\right)+e(s)\right) d s=0,
$$

which is a contradiction. Thus

$$
|x(0)|=\left|x\left(t^{* *}\right)\right|+\left|\int_{0}^{t^{* *}} x^{\prime}(s) d s\right| \leq M_{1}+M+\|N x\|_{1} .
$$


Hence

$$
\|P x\|=|x(0)| \leq \frac{\bar{\alpha}}{\bar{\beta}}\|x\|_{\infty}+\|N x\|_{1}+c_{1}
$$

where

$$
c_{1}=\max \left\{M_{1}+M, M_{1}+\frac{1}{\bar{\beta}}\left(L_{1}+\|e\|_{1}\right)\right\} .
$$

Thus we get

$$
\|x\| \leq\|P x\|+\|(I-P) x\| \leq \frac{\bar{\alpha}}{\bar{\beta}}\|x\|_{\infty}+2\|N x\|_{1}+c_{1} .
$$

From Property $\left(\mathrm{A}_{1}\right)$ we get

$$
\begin{aligned}
\|x\| \leq & \overline{\bar{\alpha}}\|x\|_{\infty}+2\|a\|_{1}\|x\|_{\infty}+2\|b\|_{1}\left\|x^{\prime}\right\|_{\infty}+2\|c\|_{1}\left\|x^{\prime \prime}\right\|_{\infty} \\
& +2\|d\|_{1}\left\|x^{\prime \prime \prime}\right\|_{\infty}+2\|g\|_{1}\left\|x^{\prime \prime \prime}\right\|_{\infty}^{\theta}+2\|r\|_{1}+2\|e\|_{1}+c_{1} \\
= & \left(2\|a\|_{1}+\frac{\bar{\alpha}}{\bar{\beta}}\right)\|x\|_{\infty}+2\|b\|_{1}\left\|x^{\prime}\right\|_{\infty}+2\|c\|_{1}\left\|x^{\prime \prime}\right\|_{\infty} \\
& +2\|d\|_{1}\left\|x^{\prime \prime \prime}\right\|_{\infty}+2\|g\|_{1}\left\|x^{\prime \prime \prime}\right\|_{\infty}^{\theta}+2\|r\|_{1}+2\|e\|_{1}+c_{1} \\
\leq & \left(2\|a\|_{1}+\frac{\bar{\alpha}}{\bar{\beta}}\right)\|x\|_{\infty}+\left(2\|b\|_{1}+2\|c\|_{1}+2\|d\|_{1}\right)\left\|x^{\prime \prime \prime}\right\|_{\infty} \\
& +2\|g\|_{1}\left\|x^{\prime \prime \prime}\right\|_{\infty}^{\theta}+2\|r\|_{1}+2\|e\|_{1}+c_{1},
\end{aligned}
$$

i.e.

$$
\begin{aligned}
\|x\| \leq & \left(2\|a\|_{1}+\frac{\bar{\alpha}}{\bar{\beta}}\right)\|x\|_{\infty}+\left(2\|b\|_{1}+2\|c\|_{1}+2\|d\|_{1} \mid\right) \mid x^{\prime \prime \prime} \|_{\infty} \\
& +2\|g\|_{1}\left\|x^{\prime \prime \prime}\right\|_{\infty}^{\theta}+2\|r\|_{1}+2\|e\|_{1}+c_{1} .
\end{aligned}
$$

It is easy to check that $\left\|x^{\prime}\right\|_{\infty} \leq\left\|x^{\prime \prime}\right\|_{\infty} \leq\left\|x^{\prime \prime \prime}\right\|_{\infty}$. Together with $\|x\|_{\infty} \leq$ $\|x\|$, it follows from the above inequality that

$$
\begin{aligned}
\|x\|_{\infty} \leq & \frac{1}{1-2\|a\|_{1}-\frac{\bar{\alpha}}{\beta}}\left[2\|b\|_{1}\left\|x^{\prime}\right\|_{\infty}+2\|c\|_{1}\left\|x^{\prime \prime}\right\|_{\infty}\right. \\
& \left.+2\|d\|_{1}\left\|x^{\prime \prime \prime}\right\|_{\infty}+2\|g\|_{1}\left\|x^{\prime \prime \prime}\right\|_{\infty}^{\theta}+2\|r\|_{1}+2\|e\|_{1}+c_{1}\right] \\
\leq & \frac{1}{1-2\|a\|_{1}-\frac{\bar{\alpha}}{\bar{\beta}}}\left[\left(2\|b\|_{1}+2\|c\|_{1}+2\|d\|_{1}\right)\left\|x^{\prime \prime \prime}\right\|_{\infty}\right. \\
& \left.+2\|g\|_{1}\left\|x^{\prime \prime \prime}\right\|_{\infty}^{\theta}+2\|r\|_{1}+2\|e\|_{1}+c_{1}\right] .
\end{aligned}
$$

Case 2. $\left|x^{\prime \prime \prime}(t)\right|>M$ for all $t \in[0,1]$. In this case from property $\left(\mathrm{A}_{2}\right)$ we obtain

$$
\begin{aligned}
\left|x^{\prime \prime \prime}\left(t_{1}\right)\right| & \leq \frac{\bar{\alpha}}{\bar{\beta}}\left|x\left(t_{1}\right)\right|+\frac{L_{1}}{\bar{\beta}}+\frac{1}{\bar{\beta}}\left|f\left(t_{1}, x\left(t_{1}\right), x^{\prime}\left(t_{1}\right), x^{\prime \prime}\left(t_{1}\right), x^{\prime \prime \prime}\left(t_{1}\right)\right)\right| \\
& \leq \frac{\bar{\alpha}}{\bar{\beta}}\|x\|_{\infty}+\frac{1}{\bar{\beta}}\left(L_{1}+\|e\|_{1}\right)
\end{aligned}
$$


so that

$$
\begin{aligned}
\left|x^{\prime \prime \prime}(t)\right| & \leq\left|x^{\prime \prime \prime}\left(t_{1}\right)\right|+\left|\int_{t_{1}}^{t} x^{(4)}(s) d s\right| \\
& \leq \frac{\bar{\alpha}}{\bar{\beta}}\|x\|_{\infty}+\frac{1}{\bar{\beta}}\left(L_{1}+\|e\|_{1}\right)+\|N x\|_{1} .
\end{aligned}
$$

Thus similarly to the above discussion, one has a $\xi \in(0,1)$ such that $x^{\prime \prime}(\xi)=0$ and there is an $\eta \in(0,1)$ such that

$$
\begin{aligned}
\left|x^{\prime \prime}(t)\right| & =\left|x^{\prime \prime}(t)-x^{\prime \prime}(\xi)\right| \\
& =\left|x^{\prime \prime \prime}(\eta)(t-\eta)\right| \\
& \leq \frac{\bar{\alpha}}{\bar{\beta}}\|x\|_{\infty}+\frac{1}{\bar{\beta}}\left(L_{1}+\|e\|_{1}\right)+\|N x\|_{1} .
\end{aligned}
$$

So we get

$$
\begin{aligned}
\left|x^{\prime}(t)\right| & =\left|x^{\prime}(t)-x^{\prime}(0)\right| \\
& \leq\left|x^{\prime \prime}(\xi)\right| \\
& \leq \frac{\bar{\alpha}}{\bar{\beta}}\|x\|_{\infty}+\frac{1}{\bar{\beta}}\left(L_{1}+\|e\|_{1}\right)+\|N x\|_{1} .
\end{aligned}
$$

From property $\left(\mathrm{A}_{3}\right)$, there is a $t^{* *} \in(0,1)$ such that $\left|x\left(t^{* *}\right)\right| \leq M_{1}$. Then, together with $(5)$,

$$
\begin{aligned}
\|P x\| & =|x(0)| \\
& =\left|x\left(t^{* *}\right)-\int_{0}^{t^{* *}} x^{\prime}(t) d t\right| \\
& \leq M_{1}+\frac{\bar{\alpha}}{\bar{\beta}}\|x\|_{\infty}+\frac{1}{\bar{\beta}}\left(L_{1}+\|e\|_{1}\right)+\|N x\|_{1} \\
& \leq \frac{\bar{\alpha}}{\bar{\beta}}\|x\|_{\infty}+\|N x\|_{1}+c_{1} .
\end{aligned}
$$

Thus

$$
\|x\| \leq\|P x\|+\|(I-P) x\| \leq \frac{\bar{\alpha}}{\bar{\beta}}\|x\|_{\infty}+2\|N x\|_{1}+c_{1} .
$$

So property $\left(A_{1}\right)$ implies

$$
\begin{aligned}
\left\|x^{\prime \prime \prime}\right\|_{\infty} \leq & \|x\| \\
\leq & \frac{2\|a\|_{1}+\frac{\bar{\alpha}}{\bar{\beta}}}{1-2\|a\|_{1}-\frac{\bar{\alpha}}{\bar{\beta}}}\left[\left(2\|b\|_{1}+2\|c\|_{1}+2\|d\|_{1}\right)\left\|x^{\prime \prime \prime}\right\|_{\infty}\right. \\
& \left.+2\|g\|_{1}\left\|x^{\prime \prime \prime}\right\|_{\infty}^{\theta}+2\|r\|_{1}+2\|e\|_{1}+c_{1}\right] \\
& +\left[\left(2\|b\|_{1}+2\|c\|_{1}+2\|d\|_{1}\right)\left\|x^{\prime \prime \prime}\right\|_{\infty}\right. \\
& \left.+2\|g\|_{1}\left\|x^{\prime \prime \prime}\right\|_{\infty}^{\theta}+2\|r\|_{1}+2\|e\|_{1}+c_{1}\right]
\end{aligned}
$$




$$
\begin{aligned}
= & \frac{1}{1-2\|a\|_{1}-\frac{\bar{\alpha}}{\beta}}\left[\left(2\|b\|_{1}+2\|c\|_{1}+2\|d\|_{1}\right)\left\|x^{\prime \prime \prime}\right\|_{\infty}\right. \\
& \left.+2\|g\|_{1}\left\|x^{\prime \prime \prime}\right\|_{\infty}^{\theta}+2\|r\|_{1}+2\|e\|_{1}+c_{1}\right] .
\end{aligned}
$$

We get (5). From (5) it follows that

$$
\left\|x^{\prime \prime \prime}\right\|_{\infty} \leq \frac{2\|g\|_{1}\left\|x^{\prime \prime \prime}\right\|_{\infty}^{\theta}+c_{1}+2\|r\|_{1}+2\|e\|_{1}}{1-2\|a\|_{1}+2\|b\|_{1}+2\|c\|_{1}+2\|d\|_{1}-\frac{\bar{\alpha}}{\bar{\beta}}}\left(1-2\|a\|_{1}-\frac{\bar{\alpha}}{\bar{\beta}}\right) .
$$

Since $\theta \in[0,1]$, there is $M_{1}^{*}>0$ such that

$$
\left\|x^{\prime \prime \prime}\right\|_{\infty} \leq M_{1}^{*}
$$

Again, it is easy to prove that

$$
\left.\begin{array}{rl}
\left\|x^{\prime \prime}\right\|_{\infty} & \leq\left\|x^{\prime \prime \prime}\right\|_{\infty} \\
\left\|x^{\prime}\right\|_{\infty} \leq\left\|x^{\prime \prime}\right\|_{\infty} & \leq\left\|x^{\prime \prime \prime}\right\|_{\infty}
\end{array}\right\} \leq M_{1}^{*}
$$

From property $\left(\mathrm{A}_{3}\right)$ we claim that there is $t^{* *} \in(0,1)$ such that $\left|x\left(t^{* *}\right)\right| \leq M_{1}$. Thus

$$
|x(t)| \leq\left|x\left(t^{* *}\right)-\int_{t}^{t^{* *}} x^{\prime}(s) d s\right| \leq M_{1}+\left\|x^{\prime}\right\|_{\infty} .
$$

Hence there is $M_{2}^{*}>0$ such that $\|x\|_{\infty} \leq M_{2}^{*}$. Hence

$$
\|x\| \leq \max \left\{\|x\|_{\infty},\left\|x^{\prime}\right\|_{\infty},\left\|x^{\prime \prime}\right\|_{\infty},\left\|x^{\prime \prime \prime}\right\|_{\infty}\right\} \leq \max \left\{M_{1}^{*}, M_{2}^{*}\right\} .
$$

Thus $\Omega_{1}$ is bounded. Let

$$
\Omega_{2}=\{x \in \operatorname{Ker} L: N x \in \operatorname{Im} L\} .
$$

For $x \in \Omega_{2}, x \in \operatorname{Ker} L$ and $Q N x=0$, thus

$$
\int_{0}^{1}(f(s, c, 0,0,0)+e(s)) d s=0, \quad \text { i.e. } \int_{0}^{1} f(s, c, 0,0,0) d s=-\int_{0}^{1} e(s) d s .
$$

Thus there is $t_{0} \in(0,1)$ such that

$$
f\left(t_{0}, c, 0,0,0\right)=-\int_{0}^{1} e(s) d s, \quad \text { so }\left|f\left(t_{0}, c, 0,0,0\right)\right| \leq\|e\|_{1} .
$$

From property $\left(\mathrm{A}_{4}\right)$ we see that there is $M^{*}>0$ such that $|c| \leq M^{*}$. Thus $\Omega_{2}$ is bounded. Next, according condition $\left(\mathrm{A}_{5}\right)$, we have the following two cases. 
Case 1. Suppose for any $c \in R$, if $|c|>M_{2}$, then $c f(t, c, 0,0,0) \leq 0$ for $t \in[0,1]$. Let

$$
\Omega_{3}=\{x \in \operatorname{Ker} L:-\lambda x+(1-\lambda) Q N x=0, \lambda \in[0,1]\}
$$

Now, similar to the proof of [6: Lemma 2.12], we prove that $\Omega_{3}$ is bounded. Suppose $x_{n}(t)=c_{n} \in \Omega_{3}$ and $\left|c_{n}\right| \rightarrow \infty$ as $n \rightarrow \infty$. Without loss of generality, suppose that $c_{n}>M_{2}$ for all $n$. Then there is $\lambda_{n} \in[0,1]$ such that

$$
\lambda_{n} c_{n}=\left(1-\lambda_{n}\right) Q N\left(c_{n}\right), \quad \text { or } \quad \lambda_{n}=\left(1-\lambda_{n}\right) \frac{Q N\left(c_{n}\right)}{c_{n}}
$$

Without loss of generality, suppose $\lambda_{n} \rightarrow \lambda_{0}$ as $n \rightarrow \infty$. Then

$$
\begin{aligned}
\left|\frac{Q N\left(c_{n}\right)}{c_{n}}\right| & =\frac{1}{\left|c_{n}\right|}\left|\int_{0}^{1}\left(f\left(s, c_{n}, 0,0,0\right)+e(s)\right) d s\right| \\
& \leq \frac{1}{\left|c_{n}\right|}\left[\|e\|_{1}+\|a\|_{1}\left|c_{n}\right|+\|r\|_{1}\right] \\
& =\|a\|_{1}+\frac{\|e\|_{1}+\|r\|_{1}}{\left|c_{n}\right|} .
\end{aligned}
$$

Thus $\frac{\mid Q N\left(c_{n}\right)}{c_{n} \mid}$ is bounded. So $\lambda_{n} \rightarrow \lambda_{0} \neq 1$ by (6). Thus, for sufficiently large $n, \lambda_{n} \neq 1$. Then

$$
\frac{\lambda_{n}}{1-\lambda_{n}}=\frac{1}{c_{n}}\left(\int_{0}^{1}\left(f\left(s, c_{n}, 0,0,0\right)+e(s)\right) d s\right) .
$$

From property $\left(\mathrm{A}_{4}\right)$, for sufficiently large $n,\left|f\left(t, c_{n}, 0,0,0\right)\right| \geq \alpha\left|c_{n}\right|$ for some $\alpha>0$. Then property $\left(\mathrm{A}_{5}\right)$ implies $f\left(t, c_{n}, 0,0,0\right)<-\alpha c_{n}$. Thus, by Fatou's Lemma,

$$
\begin{aligned}
\lim \sup & \left(\frac{1}{c_{n}} \int_{0}^{1} f\left(s, c_{n}, 0,0,0\right) d s+\frac{1}{c_{n}} \int_{0}^{1} e(s) d s\right) \\
& \leq \lim \sup \frac{1}{c_{n}} \int_{0}^{1} f\left(s, c_{n}, 0,0,0\right) d s \\
& \leq \int_{0}^{1} \lim \sup \frac{f\left(s, c_{n}, 0,0,0\right)}{c_{n}} d s \\
& \leq-\alpha \\
& <0
\end{aligned}
$$

This contradicts $\frac{\lambda_{n}}{1-\lambda_{n}} \geq 0$. Then $\Omega_{3}$ is bounded. 
set

Case 2. Suppose $|c|>M_{2}$. Then $c f(t, c, 0,0,0) \geq 0$ for $t \in[0,1]$. Indeed,

$$
\Omega_{3}=\{x \in \operatorname{Ker} L: \lambda x+(1-\lambda) Q N x=0 \text { for all } \lambda \in(0,1)\} .
$$

Like in the above argument, we can prove that $\Omega_{3}$ is bounded. In the following, we shall prove that all conditions of Theorem 1 are satisfied. Let $\Omega$ be a bounded open subset of $X$ such that

$$
\sqcup_{i=1}^{3} \bar{\Omega}_{i} \subset \Omega
$$

By Lemma $1, L$ is a Fredholm operator of index zero and $N$ is $L$-compact on $\bar{\Omega}$. By the above argument and the definition of $\Omega$, we have:

(i) $L x \neq \lambda N x$ for $(\lambda, x) \in[(\operatorname{dom} L / \operatorname{Ker} L) \cap \partial \Omega] \times(0,1)$

(ii) $N x \notin \operatorname{Im} L$ for $x \in \operatorname{Ker} L \cap \partial \Omega$.

At last, we prove that condition (iii) of Theorem $\mathrm{M}$ is satisfied. Let

$$
H(x, \lambda)= \pm \lambda x+(1-\lambda) Q N x
$$

By the definition of $\Omega$, we see that $H(x, \lambda) \neq 0$ for $x \in \partial \Omega \cap \operatorname{Ker} L$. Thus, by the homotopy property of degree, we have

$$
\begin{aligned}
\operatorname{deg}\left(Q N_{\text {Ker } L}, \Omega \cap \operatorname{Ker} L, 0\right) & =\operatorname{deg}(H(\cdot, 0), \Omega \cap \operatorname{Ker} L, 0) \\
& =\operatorname{deg}(H(\cdot, 1), \Omega \cap \operatorname{Ker} L, 0) \\
& =\operatorname{deg}( \pm \lambda I, \Omega \cap \operatorname{Ker} L, 0) \\
& \neq 0 .
\end{aligned}
$$

Thus by Theorem 1, the equation $L x=N x$ has at least one solution in $\operatorname{dom} L \cap \bar{\Omega}$. So problem (1) - (2) has at least one solution

\section{References}

[1] Aftabizadel, A. R.: Existence and uniqueness theorems for fourth-order boundary value problems. J. Math. Anal. Appl. 116 (1986), 415 - 426.

[2] Agarwal, R. P.: Focal Boundary Value Problem for Differential and Difference Equations. Dordrecht: Kluwer Acad. Publ. 1998.

[3] Agarwal, R. P., O'Regan, R. and P. J. Y. Wong: Positive Solutions of Differential, Difference and Integral Equations. Dordrecht: Kluwer Acad. Publ. 1998.

[4] Eloe, P. W.: Nonlinear eigenvalue problems for higher order Lidstone boundary value problems. Electr. J. Qual. Theory Diff. Equ. 2 (2000), $1-8$. 
[5] Eloe, P. W. and J. Henderson: Positive solutions for higher order ordinary differential equations. Electr. J. Diff. Equ. 3 (1995), $1-8$.

[6] Feng, W. and J. R. L. Webb: Solvability of three-point boundary value problems at resonance. Nonlin. Anal. - TMA 30 (1997), 3227 - 3238.

[7] Gaines, R. E. and J. L. Mawhin: Coincidence Degree and Nonlinear Differential Equations. Lect. Notes Math. 568 (1977).

[8] Graef, J. R. and B. Yang: On a nonlinear boundary value problem for fourthorder nonlinear differential equations. Appl. Anal. 72 (1999), 439 - 448.

[9] Graef, J. R. and B. Yang: Existence and non-existence of positive solutions of fourth-order nonlinear boundary value problems. Appl. Anal. 74 (2000), 201 214.

[10] Graef, J. R. and B. Yang: Positive solutions of a boundary value problem for fourth-order nonlinear differential equations. In: Proc. of Dynamics Systems and Applications 3 (2001), 217 - 224.

[11] Gupta, C. P.: Existence and uniqueness theorems for the bending of an elastic beam equations. Appl. Anal. 26 (1988), 298 - 304.

[12] Gupta, C. P.: A nonlinear boundary value problem associated with the static equilibrium of an elastic beam supported by sliding clamps. J. Math. \& Math. Sci. 12 (1989], $697-712$.

[13] Ma, R. and H. Wang: On the existence of positive solutions of fourth-order ordinary differential equations. Appl, Anal. 59 (1995), 225 - 231.

[14] Ma, R., Zhang, J. and S. Fu: The method of lower and upper solutions for fourth-order two-point boundary value problems. Math. Anal. Appl. 215 (1997), $415-422$.

[15] Mawhin, J.: Toplogical Degree Methods in Nonlinear Boundary Value Problems (NSFCBMS Regional Conference Series in Mathematics). Providence (RI, USA): Amer. Math. Soc. 1979.

[16] O'Regan, D.: Fourth (and higher) order singular boundary value problems. Nonlin. Anal. 14 (1990), 1001 - 1038.

Received 14.05.2003 